\title{
A rare case of an abnormal coeliac axis
}

\section{Abstract}

We demonstrate a rare case of an abnormal coeliac axis. The anomalies present include a long coeliac trunk and the left gastric artery branching directly from the aorta. While vascular variants are common, this particular one is quite rare. Appreciation of these variations of the coeliac axis are important for surgeons in reducing the number of complications intra operatively.

Keywords: coeliac axis, anatomical anomaly, left gastric artery, long coeliac trunk
Volume 4 Issue 3 - 2017

Jonathan Sivakumar

Discipline of Anatomy, University of Melbourne, Australia

Correspondence: Jonathan Sivakumar, Discipline of Anatomy, University of Melbourne, Carlton, VIC 3053, Australia, Tel 0413 103 472, Email Sivakumar.jonathan@gmail.com

Received: June 22, 2017 | Published: October 17, 2017

\section{Introduction}

We demonstrate a rare case of an abnormal coeliac axis. Anomalies are present in both the coeliac trunk and the left gastric artery. The coeliac artery typically has three branches - the common hepatic artery seen approaching the biliary tree, the splenic artery coursing posteriorly to the pancreas, and the left gastric artery supplying the lesser curvature of the stomach. This variant demonstrates abnormalities from the origins of the branches, while still maintaining appropriate supply to their relevant foregut derivatives in the abdomen. They do, however, have significance from a surgical perspective.

\section{Case report}

The cadaver is that of an elderly woman. Note the presence of the long coeliac trunk (Figure 1), and left gastric artery deriving directly off the aorta (Figure 2) (Figure 3). Although the cause of death in this woman is unknown, it is unlikely to be a direct result of this variant, as this anomaly still maintains functional perfusion to internal abdominal organs.

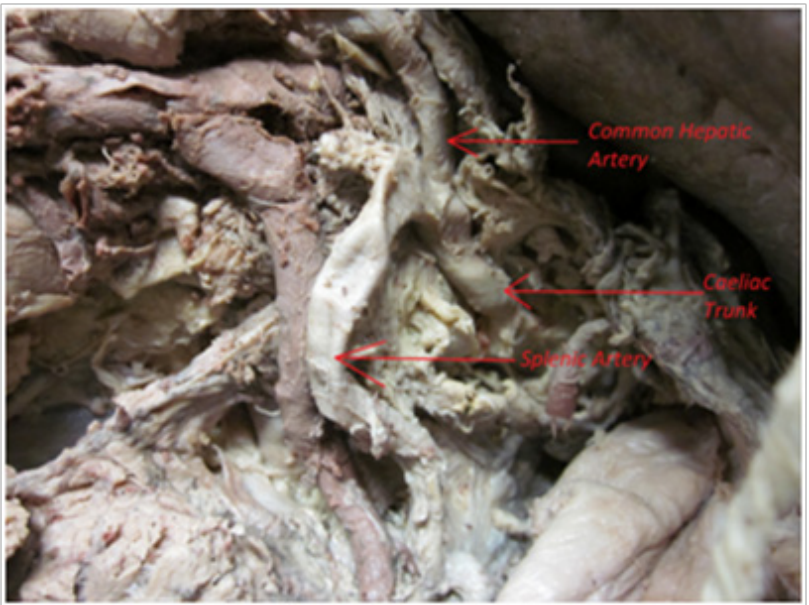

Figure I Dissection of coeliac axis demonstrating the length of the coeliac trunk.

The celiac trunk typically emerges immediately after the aortic hiatus, at the level of the T12 thoracic vertebra, as the first ventral splanchnic branch of the abdominal aorta. ${ }^{1}$ The typical length of the artery is $0.5 \mathrm{~cm}$ to $2 \mathrm{~cm}$, usually on the shorter end of this spectrum and rarely beyond this mark. ${ }^{2,3}$ In our dissection, the coeliac trunk extends further than $6 \mathrm{~cm}$. in spite of this, the patient was known to die from natural causes with pathology of the coeliac trunk not playing a role in this.

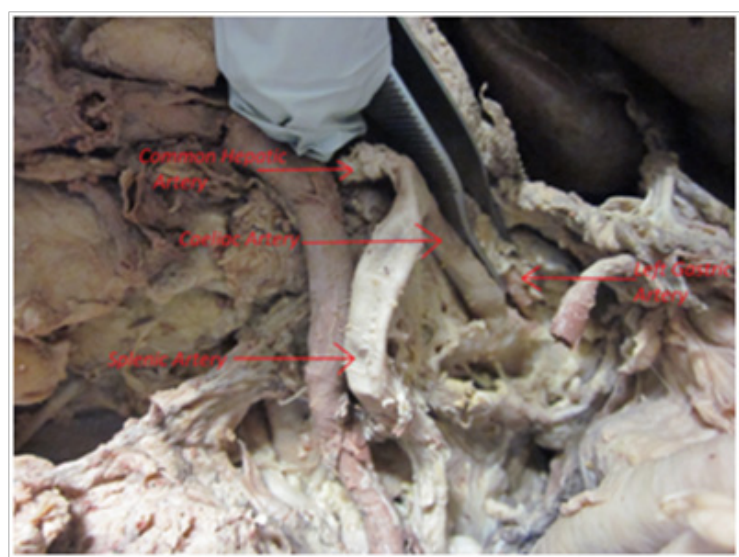

Figure 2 Dissection of coeliac axis demonstrating the origin of the left gastric artery from the abdominal aorta.

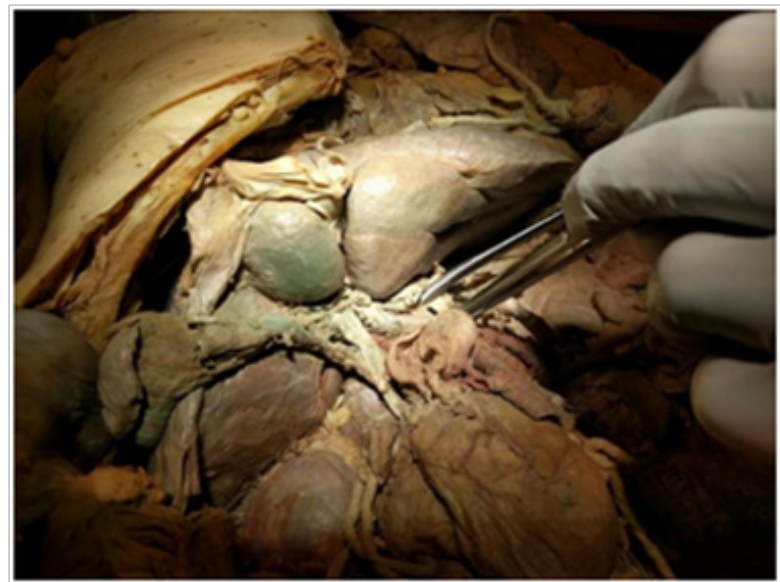

Figure 3 Overview of the cadaver with obvious anomaly noted in the anterior surface of the liver with an additional lobe. 
The branching pattern of the coeliac trunk is that it gives off the splenic artery, common hepatic artery, and the left gastric artery, which runs along the lesser curvature of the stomach. Studies on arterial variations of the abdomen showed that only $87.7 \%$ of the CT exhibited the classical trifurcation. ${ }^{4}$ Our case outlines the left gastric artery directly arising from the aorta which is a rare variant.

Appreciating the embryological process of these structures is crucial to understanding how these anomalies develop. During intrauretine life, at the four week mark, the yolk sac receives supply from a number of paired arteries in the form of vitelline arteries. ${ }^{5}$ These arteries eventually fuse to form vessels in the dorsal mesentery of the gut, later referred to as coeliac, superior mesenteric and inferior mesenteric arteries. ${ }^{5,6}$ However, the anomaly we have demonstrated in this case occurs as a result of malfusion of the vitelline arteries during the developmental stage. ${ }^{6}$

Although vascular anomalies are typically asymptomatic, appreciation of arterial variations of the coeliac trunk are important for the success of abdominal operative procedures. A solid understanding of these, could theoretically reduce unnecessary iatrogenic complications during surgery, as well ensure appropriate vessel ligation and anastomosis in made intra-operatively. ${ }^{7}$

\section{Conclusion}

This demonstration in the cadaver of an anatomical anomaly, specifically the long coeliac trunk and abnormal origin of the left gastric artery, is significant for surgeons who are commonly involved with the coeliac axis for various abdominal procedures.

\section{Guarantor of submission}

The corresponding author, myself Jonathan Sivakumar, is considered the guarantor of integrity of the manuscript as a whole.

\section{Acknowledgements}

None.

\section{Conflict of interest}

Author declares that there is no conflict of interest.

\section{References}

1. Nayak SB, Patil J, Thangarajan R, et al. Long coeliac trunk and its low origin and unusual branches: a case report. OA Case Reports. 2013;2(8):77.

2. Standring S. Gray's anatomy: the anatomical basis of clinical practice. 41st ed. New York: Elsevier Limited; 2016.

3. Malnar D, Klasan GS, Miletic D, et al. Properties of the celiac trunk-anatomical study. Coll Antropol. 2010;34(3):917-921.

4. Pamidi NVS, Vollala V. Anomalous branching pattern of coeliac trunk. International Journal of Anatomical Variations. 2008;1:8-9.

5. Sadler TW, Langman J. Langman's medical embryology. 11th ed. Baltimore: Lippincott William \& Wilkins; 2009. 385p.

6. Prakash, Rajini T, Mokhasi V, et al. Coeliac trunk and its branches: anatomical variations and clinical implications. Singapore Med J. 2012;53(5):329-331.

7. Chitra R. Clinically relevant variations of the coeliac trunk. Singapore Med J. 2010;51(3):216-219. 